\title{
Relation of Turbulence Theory to Ionospheric Forward Scatter Propagation Experiments
}

\author{
Albert D. Wheelon
}

\author{
(October 28, 1959; revised February 4, 1960)
}

\begin{abstract}
This paper attempts to relate turbulence theories to radio measurements on vhf ionospheric forward scatter circuits. To this end, the single scattering description of the electromagnetic response of electron density irregularities and the corresponding transmission expression are evaluated. Statistical distributions of signal levels are found to agree with a scattering model. The several theories for turbulent mixing of the electron density are then summarized. A turbulence mixing model is compared favorably with experimental data on absolute signal levels and their diurnal and seasonal variations. Scattering heights in the ionosphere responsible for the signals are also consistent with these theories. Frequency and distance dependence scaling laws are compared briefly with the data. The scatter signal behavior during sudden ionospheric disturbances is also explained. No attempt is made to compare meteoric and turbulence scatter contributions to the measured quantities in this paper. Approximately thirty references are given.
\end{abstract}

\section{Introduction}

The chance observation of unexplicably high field strengths beyond the optical horizon at microwave frequencies $[1]^{2}$ gave the first hint of "scatter propagation." Subsequent experiments showed that the received signal was weak but significantly greater than that predicted by round earth diffraction theory [2]. The field strength decreased slowly with distance and was observed to fade about its mean value several times a second. These observations suggested a statistical theory of scattering from turbulent fluctuations of the tropospheric refractive index $[3,4]$; a theory which has been developed and fitted to increasingly refined experiments [5].

It was inferred from the fading of ionospherically reflected skywave signals that similar refractive irregularities must also exist in the lower region $(E$ and $D$ layers) of the ionosphere. This evidence plus the reality of tropospheric scatter suggested that a similar propagation mode might be sustained at frequencies above the maximum usable frequency (MUF) by scattering from turbulent concentrations of free electrons in the ionosphere. Such a mechanism was immediately observed at vhf for ranges extending to $1,500 \mathrm{~km}$ [6], thus providing an important new mode of radio propagation.

The phenomenon of ionospheric scatter propagation has also evinced a growing scientific interest. The basic theoretical problem combines electromagnetic theory, probabilistic descriptions of random signals, and turbulence theory in an essential mixture. On the experimental side, radio waves represent an accurate, reliable tool for studying turbulent conditions at high Reynolds numbers in the ionosphere.

1 Contribution from Space Technology Laboratories, Inc., Los Angeles 45, Calif. An abbreviated version of this paper was published in J. Geophys. Research 4, 2230 (Dec. 1959).

2 Figures in brackets indicate the literature references at the end of this paper.
This paper is presented as a linkage between the turbulence theories and the radio measurements. It attempts to point up the important assumptions and questionable approximations in the present transmission expressions by way of emphasizing the accuracy of this linkage. At the same time, brief accounts of the experimental data and theoretical models are given in the interest of acquainting the radio physicists and turbulence theoreticians with the implications of the basic results in the opposite field. The present paper is preliminary in that it anticipates some results of a thoroughgoing comparison of theory and experiment for ionospheric scatter propagation, which is to be prepared shortly. This paper does not consider the contribution of small meteors to the background scatter signal.

\section{Statistical Behavior of the Signal}

Random fading of the received signal is one of the principal reasons for identifying ionospheric scatter propagation with electromagnetic scattering from turbulent irregularities. A typical time history of the amplitude $R$ of the complex voltage $x+i y$ induced in the receiver by the incident electromagnetic field is shown in figure 1. Statistical analysis of actual records show that the probability that the amplitude exceeds a given level is approximately described by a Rayleigh distribution $[7,8]$. This suggests that the process $R(t)$ is the vector amplitude of two orthogonal (phase quadrature) Gaussian random processes. On the other hand, if the received signal were produced by a large number of independent scattering contributions, one would expect the signal components to be distributed in a Gaussian manner by the Central Limit Theorem [9]. This line of reasoning indicates that scattering theory provides a reasonable (though not unique) explanation for the statistical distribution of the experimental data. 

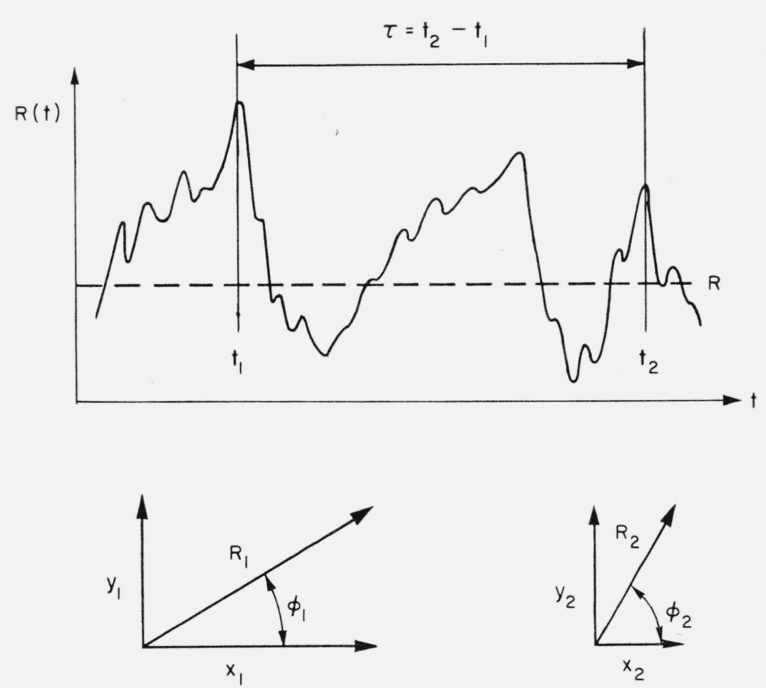

FIgURE 1. Typical amplitude versus time record of ionospheric scatter propagation signal amplitude together with corresponding time - displaced vector voltage diagrams which are assumed to describe the process.

To be more precise about these ideas, it is important to set down their corresponding analytical expressions. The joint probability distribution for the Gaussian random processes $x(t)$ and $y(t)$ is,

$P(x, y) d x d y=\frac{d x d y}{2 \pi \sigma^{2} \sqrt{1-\rho^{2}}} \exp -\left[\frac{x^{2}+y^{2}-2 \rho x y}{2 \sigma^{2}\left(1-\rho^{2}\right)}\right]$

where

$$
\begin{aligned}
& \left\langle x^{2}\right\rangle=\left\langle y^{2}\right\rangle=\sigma^{2} \\
& \langle x y\rangle=\sigma^{2} \rho .
\end{aligned}
$$

When eq (2.1) is transformed to the polar coordinates $(R, \phi)$ of figure 1 , and the phase averaged out, the amplitude distribution becomes:

$$
\begin{aligned}
& P(R) R d R=\frac{R d R}{\sigma^{2} \sqrt{1-\rho^{2}}} I_{o}\left(\frac{R^{2}}{2 \sigma^{2}} \frac{\rho}{1-\rho^{2}}\right) \\
& \exp -\left[\frac{R^{2}}{2 \sigma^{2}\left(1-\rho^{2}\right)}\right] .
\end{aligned}
$$

This expression reduces to the Rayleigh distribution if the component correlation $\rho$ is assumed to be zero. However, this assumption is probably not justified for electromagnetic scattering - as it is for electrical shot noise - and the more general expression (2.2) may explain interesting anomalies in the experimental envelope distribution data [10]. The data generally seem to emphasize larger values of $R$ (relative to the mean) more than the Rayleigh distribution predicts, with corresponding reductions in the occurrence of small values. This behavior could also be explained by: (1) Admixture of a steady signal componentperhaps due to partial ionospheric reflections above the MUF, or (2) trends in the average signal, which would produce larger apparent deviations from the mean level when viewed over a finite data sample. On the other hand, such anomalies may represent the effect of meteor-reflected signals.

One can also discuss the joint amplitude distribution for two amplitude processes $R_{1}$ and $R_{2}$ [5]. These could refer to signals received at two adjacent aerials, such as are often used in diversity receiving systems. $R_{1}$ and $R_{2}$ could also refer to two timedisplaced records of the same circuit signals, as shown in figure 1. Dual process distributions introduce additional statistical parameters $\mu$, which represent the (time, space, etc.) correlations between the component processes $x_{1}, y_{1}, x_{2}, y_{2}$. The purely probabilistic predictions of such distributions have been confirmed on tropospheric scatter circuits [11] but have yet to be studied carefully for ionospheric scatter.

In general, one can say that the statistical predictions of scattering theory are consistent with the experimental data. This agreement is neither exact nor unique, but it is satisfactory. The real problem in scatter theory is to predict successfully the statistical parameters, $\sigma, \rho, \mu$, etc., as functions of the variables which are experimentally controlled: radio frequency, path distance, time of day and season, etc. No theoretical estimates for the correlation coefficients $\rho$ and $\mu$ have been published, with the exception of certain calculations for tropospheric scatter [5], which may or may not be applicable to iono spheric circuits. Research to date has been focused exclusively on the average power, which according to eq $(2.2)$ is

$$
<R^{2}>=2 \sigma^{2}
$$

\section{Electromagnetic Scattering by Turbulen Irregularities}

The basic wave equation which discribes radio wave propagation through an ionized plasma of elec tron density $N_{0}$ with stochastic fluctuations $\boldsymbol{\delta} \mathbf{N}$ addec thereto is:

$$
\left[\nabla^{2}+k^{2}-r_{0}\left(N_{0}+\boldsymbol{\delta} \boldsymbol{N}\right] \vec{E}=0,\right.
$$

where $k=2 \pi / \lambda$ is the free space wavenumber anc $r_{0}=2.8 \times 10^{-13} \mathrm{~cm}$ the classical electron radius. Th mean electron density $N_{0}$ in (3.1) is usually omitted since refractive effects have been found to be negli gible [12]. In establishing expression (3.1), one as

sumes that: (1) The stochastic variations $\delta \mathbf{N} \overrightarrow{(r}, t$ rearrange themselves in a time which is long com pared to the period of the radio oscillations, and $(2$ that gradients of $\boldsymbol{\delta} \mathbf{N}$ are negligible over a wavelength The latter assumption is clearly the more question able.

It is not possible to solve eq (3.1) exactly, becaus $\boldsymbol{\delta} \mathbf{N}(r, t)$ is an unknown stochastic function of positio and time. Heretofore, the Born approximation ha 
been used exclusively to describe single scattering by such fluctuations. 'This is essentially a three-dimensional iterative solution of the wave eq (3.1), which gives the scattered field strength at the receiver $R$ in terms of the unperturbed wave $E_{0}(r)$.

$$
E_{s}(R)=r_{o} \int_{V} \mathrm{~d}^{3} r G(R, r) \boldsymbol{\delta} \mathbf{N}(r, t) E_{o}(r),
$$

where

$$
G(R, r)=\frac{e^{i k}|\overrightarrow{R-r}|}{4 \pi|\overrightarrow{R-r}|}
$$

and

$$
E_{o}(r)=\sqrt{\frac{P_{T} G_{T}}{4 \pi}} \frac{e^{i k_{1} \cdot \vec{r}}}{r}
$$

$P_{T}$ is the transmitted power and $G_{T}$ the transmitter gain function. The integration of (3.2) is extended over the common volume $V$ illuminated by both the transmitter and receiver.

Since both the transmitter and receiver are usually many wavelengths from the scattering blobs in the common volume, one can use the far-field approximation [5] to express the scattered field strength as:

$$
E_{s}(R) \simeq r_{0} \sqrt{\frac{P_{T}}{4 \pi}} \frac{e^{i k R_{2}}}{4 \pi} \int_{V} d s \frac{e^{\left.\overrightarrow{i\left(k_{2}-k_{1}\right.}\right) \cdot r}}{R_{1} R_{2}} \delta \mathbf{N}(r, t) \sqrt{G_{T}}
$$

where the various quantities are defined in figure 2 . The orthogonal random signal component $x(t)$ and $y(t)$ of figure 1 are identified with the real and imaginary parts of this expression respectively. The received power is computed from this expression by averaging its square and multiplying with the effective receiver area. In terms of the scattering difference vector $\vec{K}=\overrightarrow{k_{1}-k_{2}}$ :

$P_{R}=P_{T} r_{0}^{2} \frac{A_{R}}{(4 \pi)^{3}} \int d^{3} r \int d^{3} r^{\prime} \frac{\left(G_{T} G_{T}^{\prime} G_{R} G_{R}^{\prime}\right)^{1 / 2}}{R_{1} R_{1}^{\prime} R_{2} R_{2}^{\prime}} e^{\left(\overrightarrow{i r} \cdot \vec{K}-\overrightarrow{r^{\prime}} \cdot \vec{K}^{\prime}\right)}$

$$
\times<\boldsymbol{\delta} \mathbf{N}(r, t) \boldsymbol{\delta} \mathbf{N}\left(r^{\prime}, t\right)>\text {, }
$$

where $A_{R}$ is the actual receiver area. The space correlation probably vanishes unless $r$ and $r^{\prime}$ are within several kilometers of one another, and the other factors are relatively weak functions of position, so that $R_{1}=R_{1}^{\prime}, R_{2}=R_{2}^{\prime}$, etc., to a good approximation. Transforming the double integral to sum and difference coordinates $(r, \rho)$, one finds:

$$
\begin{aligned}
P_{R}= & P_{T} r_{0}^{2} \frac{A_{R}}{(4 \pi)^{3}} \int_{V} d{ }^{3} r \frac{G_{T} G_{R}}{R_{1}^{2} R_{2}^{2}} \int d^{3} \rho e^{\overrightarrow{i K} \cdot \vec{\rho}} \\
& <\boldsymbol{\delta} \mathbf{N}(\vec{r}, t) \boldsymbol{\delta} \mathbf{N}(\overrightarrow{r+\rho, t})> \\
= & P_{T} r_{0}^{2} \frac{A_{R}}{(4 \pi)^{3}} \int_{V} d^{3} r \frac{G_{T} G_{R}}{R_{1}^{2} R_{2}^{2}} S(\vec{K}) .
\end{aligned}
$$

where $S(K)$ is the spectrum of turbulent irregularities to be discussed presently. This expression is probably a valid approximation, at least insofar as single scattering is the dominant scattering mechanism.

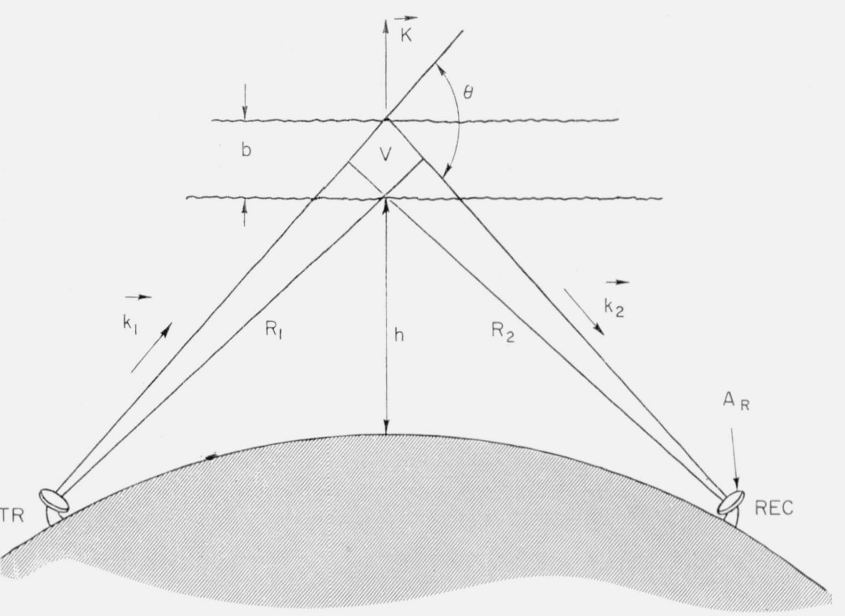

Figure 2. Geometry for analysis of electromagnetic scattering from ionospheric irregularities.

Transmission expressions which have been used thus far in interpreting experimental data acquired on forward scatter communication circuits, however, represent further assumptions, which are well worth identif ving.

a. The ionospheric irregularities are assumed to be isotropic, ${ }^{3}$ so that the spectrum $S(K)$ appearing in (3.7) depends only on the magnitude of the scattering difference vector.

$$
K=|\vec{K}|=\frac{4 \pi}{\lambda} \sin \left(\frac{\theta}{2}\right)
$$

where $\theta$ is the scattering angle between the upgoing and downgoing rays at the integration point $r$. The scattering process thus acts like a narrow band filter on the spectrum, emphasizing the uniquely important wavenumber given by eq (3.8). The isotropy assumption evidently precludes a discussion of magnetically-alined irregularities with the resulting transmission expression.

b. The volume integration $\left(d^{3} r\right)$ is collapsed by multiplying $V$ by an average value of the integrand. The average scattering angle $\bar{\theta}$ is usually chosen to be that at the path midpoint, corresponding to the strongest scattering contribution (i.e., smallest $K$ ). The effective volume is set by $\bar{\theta}$, the transmitter gain, and the width of an effective scattering layer $b$ (see fig. 2):

$$
V=\frac{4 \pi}{G_{T}} R_{1}^{2} b \csc \frac{\bar{\theta}}{2} .
$$

${ }^{3}$ Anisotropic correlation functions were used by Staras in his extensive analysis of tropospheric scatter circuits and by Booker in discussing the backscattering from freld-alined auroral irregularities. However, these techniques have not been applied to ionospheric forward scattering to date. 
c. Polarization effects have been neglected, although a relatively simple theory predicts a factor $\sin ^{2} \chi$ in eq (3.6), where $\chi$ is the angle between $\vec{E}_{0}$ and the scattering direction $\vec{k}_{2}$. More precise accounts $[13,14]$ of the vector field response support this approximation.

The resulting transmission equation,

$$
P_{R}=P_{T} r_{0}^{2} A_{R} G_{R} \frac{b}{R_{2}^{2}} \csc \left(\frac{\vec{\theta}}{2}\right) S\left(\frac{4 \pi}{2} \sin \frac{\bar{\theta}}{2}\right)
$$

has formed the only linkage between turbulence theory and experiments thus far. Expression (3.10) is evidently a substantial approximation to the actual electromagnetic response of the irregularities, and it is well to be aware of its limitations. On the other hand, a new technique has been developed [5] which allows one to remove all of the above approximations - within the framework of single scattering - at the expense of complicated integrations.

The scattering cross section (per unit volume per unit solid angle) concept is often employed, viz,

$$
\sigma(\theta, \lambda)=r_{0}^{2} S\left(\frac{4 \pi}{\lambda} \sin \frac{\bar{\theta}}{2}\right)
$$

However, this approach does not emphasize the joint frequency-distance dependence of (3.10) which is characteristic of scatter propagation. In any case, it is quite clear that the spectrum of irregularities is the basic description of the turbulent electron density variations which is required by the electromagnetic theory.

\section{Turbulence Theories}

Results of the last section show that the spectrum of turbulent electron irregularities is the physical quantity of central interest for scatter propagation. More particularly, one is interested in the spectrum at wavelengths

$$
K=\frac{4 \pi}{\lambda} \sin \frac{\bar{\theta}}{2}
$$

which lie in the following range for typical vhf scatter paths.

$$
0.2 \mathrm{~m}^{-1}<K<1.3 \mathrm{~m}^{-1},
$$

On the other hand, the wavenumber $K_{s}$ at which diffusion-viscosity effects ought to become important is within - or at least near - the range of interest.

$$
K_{s}=\left(\frac{V_{0}^{3}}{l_{0} \nu^{3}}\right)^{1 / 4} \simeq 0.5 \mathrm{~m}^{-1}
$$

In terms of the spectral ranges identified in figure 3, this means that one is probably interested in the spectrum for the difficult transition region between the inertial subrange and the asymptotic dissipa-

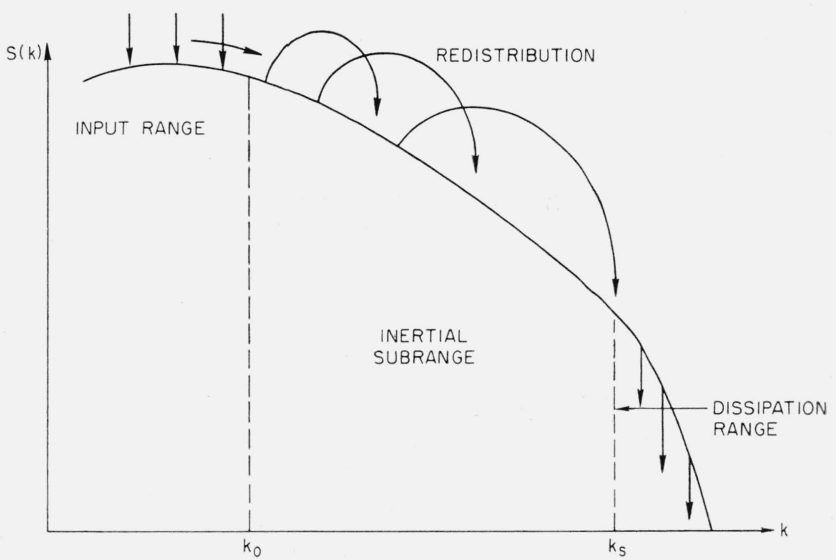

Figure 3. Typical spectrum of dielectric irregularities plotted logarithmically versus wavenumber, identifying various turbulence ranges.

tion range. This is unfortunate, since one probably cannot use the dimensional analysis methods which have proven so powerful in the inertial subrange.

To avoid notational confusion, we use $S(K)$ to denote the three-dimensional spectrum, which is defined as the Fourier transform of the space correlation function.

$$
\langle\boldsymbol{\delta} \mathbf{N}(\bar{r}, t) \boldsymbol{\delta} \mathbf{N}(\overrightarrow{r+R}, t)\rangle=\frac{1}{8 \pi^{3}} \int d^{3} k e^{\overrightarrow{i k} \cdot \vec{R}} S(\vec{K})
$$

If the turbulent irregularities are isotropic, the spectrum depends only on the magnitude of the vector wavenumber $\vec{k}$, and the entire description is much simplified.

The one-dimensional spectrum is denoted by $\Gamma(k)$, and corresponds to wavenumber contributions to the mean square variation.

$$
\left\langle\delta N^{2}\right\rangle=\frac{1}{2 \pi^{2}} \int_{0}^{\infty} d k k^{2} S(\vec{K})=\int_{0}^{\infty} d k \Gamma(k) .
$$

This establishes the relation:

$$
S(\vec{K})=2 \pi^{2} \frac{\Gamma(k)}{k^{2}} .
$$

Three distinct physical theories have been developed in recent years to describe the irregularities of electron density established by the turbulent velocity field of neutral ionospheric gases:
a. Theory of pressure fluctuations,
b. Obukhov-Corrsin mixing theory,
c. Villars-Weisskopf mixing theory.

Each of these theories has now been extended into the dissipation regime, and the essential results are tabulated for convenience in figure 4, together with brief references to the many contributors. Without 


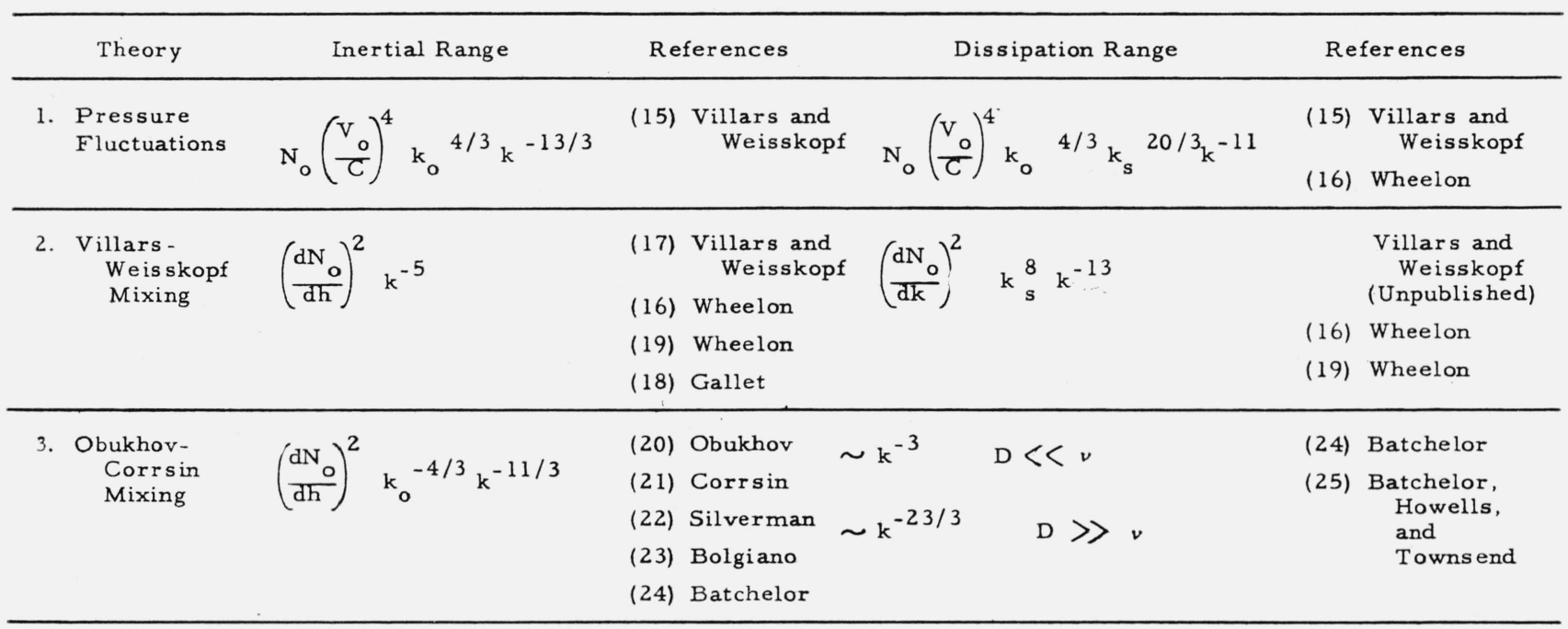

Figure 4. Summary of spectral models $S(k)$ for electron density irregularities in the lower ionosphere.

attempting to plead one case or another in the colorful but unfortunate controversy which has been kindled in this new field, it is important to note several points which now seem rather clearcut:

a. The theory of pressure fluctuations has essentially been abandoned as a major source of ionospheric irregularities $[17,18]$.

b. The two mixing theories both agree that a (random) convective mixing-diffusion equation of the form:

$$
\left(\frac{\partial}{\partial t}-D \nabla^{2}\right) \delta N(r, t)+\vec{v}(r, t) \cdot \vec{\nabla} \delta N(r, t)=\vec{v} \cdot \vec{\nabla} N_{0}
$$

characterizes the process, where $\vec{v}(r, t)$ is the turbulent solution of the Navier-Stokes equations.

c. The Obukhov-Corrsin theory is patterned precisely on the Kologomoroff theory of the velocity spectrum. It essentially disregards the differential eq (4.5), assumes that the input and inertial range do not overlap, and arrives at the following result by dimensional analysis:

$$
\Gamma(k) \sim k^{-5 / 3}\left(\frac{d N_{0}}{d h}\right)^{2} k_{0}^{-4 / 3} .
$$

d. Villars and Weisskopf noted that the gradient of $N_{0}$ appears in the basic eq (4.5). Accepting $d N_{0} / d h$ as a legitimate parameter for dimensional analysis, one finds:

$$
\Gamma(k) \sim k^{-3}\left(\frac{d N_{0}}{d h}\right)^{2}
$$

e. In deriving expressions for the transition and dissipation ranges, both mixing theories use approximate analysis which are open to question.

f. None of the theories discussed here have as yet been extended to include the influence of the earth's magnetic field.
The reader is referred to the basic references indicated in figure 4 for detailed arguments and precise expressions.

\section{Signal Level and Scattering Heights}

One can combine the approximate transmission expression (3.10) with various turbulence models listed in figure 4 to predict theoretically the received power on a particular scatter link. This calculation and comparison has been performed for ionosphere forward scatter circuits so far $[17,26]$ only for the Villars-Weisskopf mixing model (4.7), which is subject to a rather stringent test since it contains no adjustable turbulence parameters. Using only the inertial subrange result for illustration, one has for the theoretical transmission equation:

$$
P_{R}=P_{T} \frac{b A_{R} G_{R}}{R_{2}^{2}} 2 \pi^{2} r_{0}^{2}\left(\frac{\lambda}{4 \pi}\right)^{5} \csc ^{6}\left(\frac{\theta}{2}\right)\left(\frac{d N_{0}}{d h}\right)^{2} .
$$

All of the factors in this equation are known for a given circuit, except the appropriate value of electron density gradient. This evidently depends on the ambient electron density profile at the common volume altitude.

In figure 5 we reproduce a suggested typical noontime profile of electron density for the lower ionosphere from reference [26]. The sharp lower boundary at $70 \mathrm{~km}$ is consistent with vlf reflection data, and the upper portions agree moderately well with rocket data. According to both mixing theories, large gradient portions of the profile ought to give rise to more intense turbulent irregularities and hence stronger scattering. A sharp gradient at 70 $\mathrm{km}$ would reproduce the strong daytime signal contribution. The power levels measured on the Cedar Rapids-Sterling path [7] require that this 


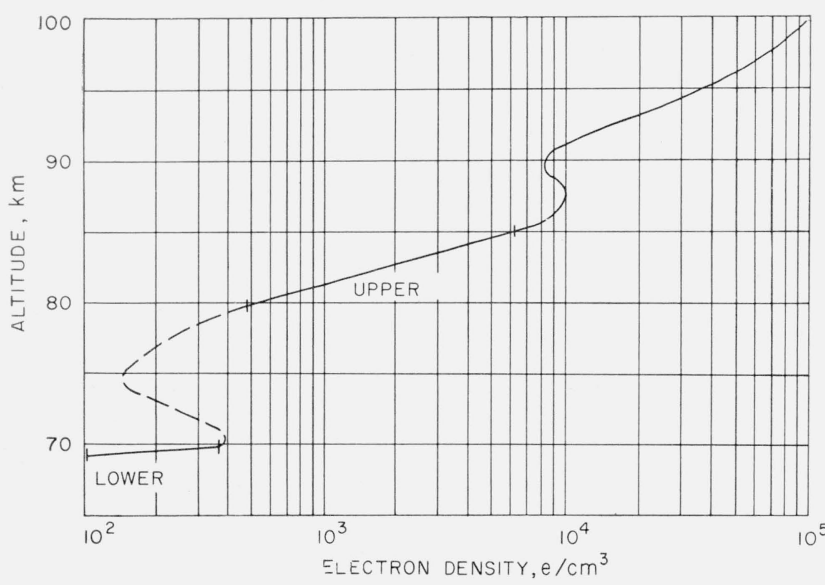

Figure 5. Possible noontime profile of electron density in the lower ionosphere which is consistent with various radio measurements from reference [26].

gradient be approximately

$$
\left.\frac{d N_{0}}{d h}\right|_{70} \simeq 2,000 \frac{e}{\mathrm{~cm}^{3}} \text { per } \mathrm{km},
$$

which represents a very fast rise to the modest total electron density at this height. It would be interesting to see if the Obukhov-Corrsin theory gives a smaller number.

A weaker gradient at approximately $85 \mathrm{~km}$ is suggested in figure 5, and should also contribute to the scatter signal. The gradient at this height is thought to be

$$
\left.\frac{d N_{0}}{d h}\right|_{85} \simeq 3 \mathrm{C} 0 \frac{e}{\mathrm{~cm}^{3}} \text { per } \mathrm{km},
$$

corresponding to a signal some 10 - to 15 - $\mathrm{db}$ weaker than the $70-\mathrm{km}$ contribution. It is interesting that both of these signal components are found in the short pulse measurements of Pineo [27]. In general, he finds unequal signals from two levels separated by about $15 \mathrm{~km}$. Further evidence for weaker scattering from an upper layer comes from the long range NBS experiments [28], which compared 36-Mc signals over $2270 \mathrm{~km}$ with $50-\mathrm{Mc}$ signals received on a $1240-\mathrm{km}$ path. Earth screening on the longer path obscured all volume elements below $87 \mathrm{~km}$ in these experiments, and the (frequency-scaled) received signal was some 10-db weaker than the shorter circuit, which presumably could exploit both the 70 - and $85-\mathrm{km}$ ranges. Further evidence for this dual stratification comes from the vertical incidence pulse backscatter measurements made at hf (see [26] for references).

\section{Diurnal and Seasonal Variations}

The signal level of a vhf scatter circuit exhibits large changes during each day, year, and sunspot cycle. Experimental data for the NBS 50-Mc Cedar Rapids-Sterling circuit [7] is shown in figure 6, to- gether with the long range Newfoundland-Azores path. The first record is typical of most vhf paths and shows a 10-db rise from morning to noon, followed by a 15-db drop to postsunset values. The corresponding meteor count is strongly peaked at 0600 and is essentially negligible between noon and midnight. This suggests that the afternoon and early evening is under strong solar control.

A theoretical explanation [26] for the afternoon signal drop is offered by turbulent mixing theories through the mean profile gradient factors, since the mean profile is under very strong solar control all day. The noontime signal is contributed by the $70-\mathrm{km}$ level (sec. 5) and the afternoon decay shown in figure 6 ought to reflect the way in which this sharp gradient is erased by recombination at sunset. The rapid drop observed corresponds to the very fast recombination rates at lower levels. On the other hand, the decay of the upper gradient is found to be much more gradual, and in fact should produce measurable scatter signals all night. The noon-toevening $1500-\mathrm{km}$ signal drop in figure 6 presumably corresponds to the shift of scattering height from the 70 - to $85-\mathrm{km}$ levels, which is confirmed by pulse measurements [27]. The comparative absence of a steep signal drop on the long range path is again consistent with a slow recombination of the upper gradient. This line of reasoning forms quite a satisfactory explanation for the existing data on diurnal variations. The basic key to this agreement is the relationship between turbulent intensity and the mean profile provided by the mixing theories.

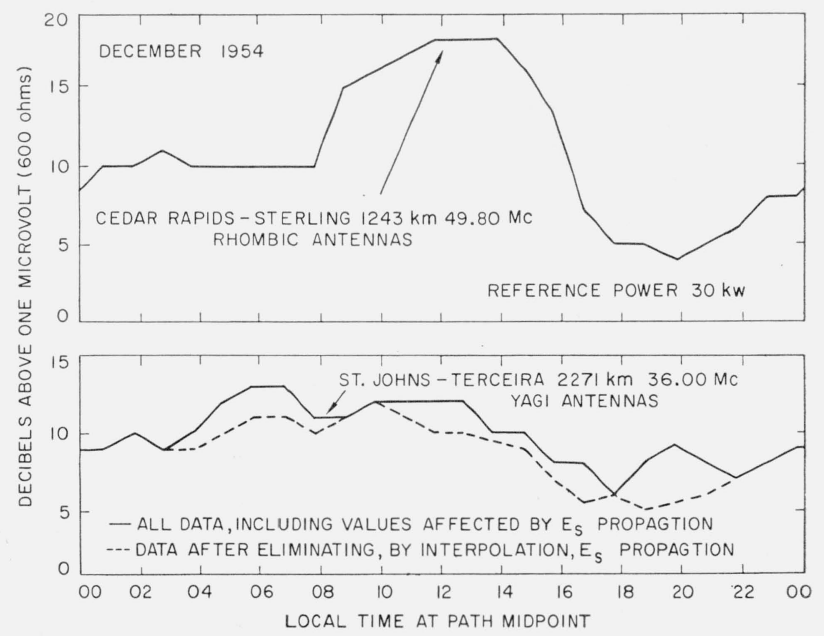

FiguRE 6. Diurnal variation of power on two vhf scatter paths from reference [\%].

Recent published data [29] on correlation of vhf scatter signal strength and sunspot number demonstrates a strong solar control over a $6-y$ r period. A good explanation is again provided by the relationship between turbulence intensity and the mean profile (gradient), since the latter is clearly under consistent solar control. 


\section{Frequency Dependence}

The basic transmission eq (3.10) depends upon the radiofrequency through: (1) The turbulence spectrum $S(K)$, and (2) the effective receiving area $A_{R} G_{R}$. To avoid confusion on how the second effect is accounted for, it is convenient to compare received power on two different frequencies for scaled antennas, viz, equal $G_{R}$ factors. The definitive experiments by the NBS group [10] on frequency dependence actually used carefully scaled rhombic antennas, so as to insure identical illumination of the scattering layers and similar antenna response. With this convention, the frequency variation is that due to the spectrum plus a $\lambda^{2}$ factor which representsthe physical scaling of the receiving aerial size. If the spectrum is expressible as a simple inverse power $(n)$ of wavenumber in the relevant turbulent range, one would predict a (scaled antenna) frequency scaling law [30],

$$
P_{R}=\frac{\text { const }}{f^{n+2}}
$$

The most recent experimental data is that reported by Blair [10], using 30, 40, 50, 74, and $108 \mathrm{Mc}$. $\mathrm{He}$ finds that the exponent $n$ in eq (7.1) is variable, ranging from 7 to 10 . This variation is both diurnal and seasonal, so that it is dangerous to quote a mean value. During late afternoon hours, however, when scattering from turbulence is presumably the dominant mode, the exponent $n$ is definitely higher, and a value of 8 to 9 may be representative. Comparing this with the turbulence results tabulated in figure 4, the Obukhov-Corrsin inertial range result gives $f^{-5.6}$, which is definitely too low. The VillarsWeisskopf inertial result $f^{-7}$ is occasionally correct, but the corresponding dissipation behavior $f^{-15}$ is too high. The $f^{-9.6}$ dissipation result for $d>>\nu$ given by Batchelor, Howells, and Townsend [25] describes some of the records. On the balance, however, one finds that none of the theories give a satisfactory explanation for the important frequency exponent variability.

Earlier experiments of frequency dependence [7,31] indicated a higher exponent scaling law between 50 and $100 \mathrm{Mc}$, than that measured between 30 and $50 \mathrm{Mc}$. Because the scattering process acts like a narrow band filter on the spectrum (sec. 3), it was suggested that the viscosity transition wavenumber of the ionosphere (4.2) did, in fact, divide the wavenumber range of experimental interest (4.1). The steeper slope approaching the dissipation range and the smaller inertial slope seemed to explain the data rather nicely [16]. However, the newer data [10] indicates that such a dichotomy does not exist, and that the exponent is constant over the wavenumber range employed at any given hour - even though the slope itself shows diurnal and seasonal variations.

\section{Distance Dependence}

If the turbulent spectrum function $S(K)$ is expressible as a simple inverse power $(n)$ of wavenumber in the relevant range, two factors which depend on path length $d$ occur in the basic transmission eq (3.10),

$$
P_{R}=\frac{\text { constant }}{R_{2}^{2}\left(\sin \frac{\theta}{2}\right)^{n+1}}
$$

If a denotes the mean radius of the earth, the distance $R_{2}$ from the average scattering point to the receiver is given by

$$
R_{2}^{2}=a^{2}+(a+h)^{2}-2 a(a+h) \cos \left(\frac{d}{2 a}\right) \simeq\left(\frac{d}{2}\right)^{2} \text { for } a \gg d .
$$

The scattering angle is a more complicated function of distance $d$ and scattering height $h$ [16].

$$
\tan \frac{\theta}{2}=\frac{1-\cos \left(\frac{d}{2 a}\right)+\frac{h}{a}}{\sin \left(\frac{d}{2 a}\right)} \simeq \frac{d}{4 a}+\frac{2 h}{d} \text { for } d \ll a .
$$

A graphical evaluation of $\Theta$ versus $d$ for various $h$ is given in reference [16]. By combining all of the above expressions, it is possible to establish the variation of received power with distance predicted by any turbulence model $(n)$. However, one should also note that earth screening horizon limitations have the effect of raising the lowest scattering midpoint as the distance is increased. Insofar as the strongest scattering centers are located at the low er height (sec. 6), this vertical inhomogeneity must be reflected in an additional distance dependence of the mean signal level.

The experimental data now available is probably not accurate enough for a decisive test of the theories. The early data [7] used successive measurements of $50-\mathrm{Mc}$ signals at 491, 592, and $811 \mathrm{~km}$, whereas one would have preferred simultaneous data over longer paths. The shipborne DSIR experiments [31] on $41 \mathrm{Mc}$ gave limited results in terms of defining the distance scaling law [30]. Recent (unpublished) airborne receiver experiments made by the Lincoln Laboratory group out to $3000 \mathrm{~km}$ on $50 \mathrm{Mc}$ should provide a valuable basis for testing the various theoretical results.

\section{Sudden Ionospheric Disturbances}

A sudden increase in the flux of ionizing radiation from the sun increases the electron density rather quickly in the lower ionosphere, which in turn increases the nondeviative absorption of radio waves which pass through the $D$ region [33]. This prompt absorption is called a sudden ionospheric disturbance, and affects both totally reflected short wave (hf) signals and the partially reflected waves responsible for (vhf) ionospheric scatter propagation. A typical set of simultaneous signal amplitude records made on a shortwave circuit at $6 \mathrm{Mc}$ and two adjacent scatter links at 28 and $50 \mathrm{Mc}$ is reproduced in figure 7 , from reference [7]. The shortwave link drops out immediately, while the $50-\mathrm{Mc}$ scatter link increases 


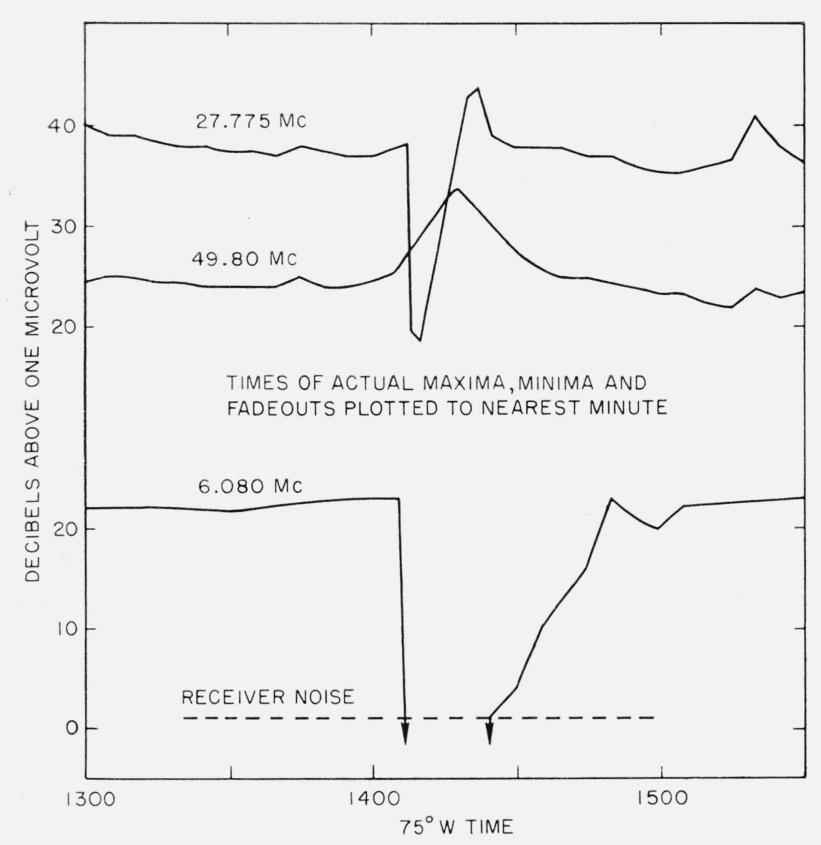

Figure 7. Transient behavior of power on three frequencies taken from reference [\%].

gradually until the recovery cycle of the transient $D$ region begins. The $28-\mathrm{Mc}$ link shows a mixture of the two effects. Viewed in a qualitative way, these data lend strong support to the notion that ionosphereic scatter links are under direct solar control much of the time [29].

One can construct ${ }^{4}$ an analytical explanation for the transient amplitude behavior shown in figure 7 . Nondeviative absorption of a radio wave of frequency $f$ can be represented approximately by a multiplicative factor [33];

$$
\exp -\frac{\gamma}{f^{2}} \int_{0}^{h} d y N(y, t) \nu(y)
$$

where $h$ is the reflection height. $N(y, t)$ is the instantaneous value of electron density at the ray path integration point $y$, and $\nu$ is the collision frequency at the same point. The totally reflected shortwave field strength is modified by the factor (9.1) alone, which is very large because the carrier frequency $f$ is relatively small. The rapid realization of this absorption loss indicated by figure 7 indicates how rapidly the electron density profile is modified by the burst of solar ionization.

If one is to believe the turbulent mixing theories, the field strength of an ionospheric scatter circuit depends both on the transient electron density profile and its gradient, viz,

$$
P \simeq\left[\frac{d N(h, t)}{d h}\right]^{2} \exp -\frac{\gamma}{f^{2}} \int_{0}^{h} d y N(y, t) \nu(y)
$$

\footnotetext{
${ }^{4}$ A. D. Wheelon, unpublished calculations.
}

Because of height-dependent recombination rates, etc., rapid changes in the flux of ionizing radiation produce substantial modification of the basic electron density height profile. Careful analysis shows that transient modification of ionization gradients in the $D$ region can explain the signal increase of 50 Me shown in figure 7. (The exponential factor is negligible at such high frequencies.) On the other hand, the $28-\mathrm{Mc}$ signal ought to suffer significant absorption, and the competition between enhanced gradient scattering and this absorption can be explained with the theory. A possible explanation for this type of behavior is thus provided by turbulent mixing theories and standard absorption expressions.

\section{References}

[1] K. Bullington, Characteristics of beyond-the-horizon radio transmission, review paper, Proc. IRE 43, 1175 (1955).

[2] H. Bremmer, Terrestrial radio waves (Elsevier, New York, N.Y., 1949).

[3] H. G. Booker and W. E. Gordon, A theory of radio scattering in the troposphere, Proc. IRE 38, 401 (1950)

[4] C. L. Pekeris, Note on the scattering of radiation in an inhomogeneous medium, Phys. Rev. 71, 268 (1947).

[5] A. D. Wheelon, Radiowave scattering by tropospheric irregularities, J. Research NBS 63D, 205 (1959).

[6] D. K. Bailey, R. Bateman, L. V. Berkner, H. G. Booker G. F. Montgomery, E. M. Purcell, W. W. Salisbury, and J. B. Wiesner, A new kind of radio propagation at very high frequencies observable over long distances, Phys. Rev. 86, 141 (1952)

[7] D. K. Bailey, R. Bateman, and R. C. Kirby, Radio transmission at vhf by scattering and other processes in the lower ionosphere, Proc. IRE 43, 1181 (1955).

[8] W. G. Abel, J. T. deBettencourt, J. H. Chisholm, and J. F. Roche, Investigations of scattering and multipath properties of ionospheric propagation at radio frequencies exceeding the MUF, Proc. IRE 43, 1255 (1955).

[9] R. A. Silverman, Some remarks on scattering from eddies, Proc. IRE 43, 1253 (1955).

[10] J. C. Blair, Frequency dependence of vhf ionospheric scattering, unpublished work (1959).

[11] H. Staras, Diversity reception with correlated signals, J. Appl. Phys. 27, 93 (1956). See also Proc. IRE 44, 1057 (1956), and Study and investigation of tropospheric scattering, RCA Rept. Pt. A, p. 91 (1956).

[12] A. D. Wheelon, Refractive corrections to scatter propagation, J. Geophys. Research 62, 343 (1957).

[13] M. Balser, Some observations on scattering by turbulent irregularities, Trans. IRE PGAP AP-5, 383 (1957).

[14] S. Stein, Some observations on scattering by turbulent irregularities, Trans. IRE PGAP AP-6, 299 (1958).

[15] F. Villars and V. F. Weisskopf, The scattering of electromagnetic waves by turbulent atmospheric fluctuations, Phys. Rev. 94, 232 (1954).

[16] A. D. Wheelon, Radio frequency and scattering angle dependence of ionospheric scatter propagation at vhf, J. Geophys. Research 62, 93 (1957).

[17] F. Villars and V. F. Weisskopf, On the scattering of radio waves by turbulent fluctuations of the atmosphere, Proc. IRE 43, 1232 (1955).

[18] R. M. Gallet, Aerodynamical mechanisms producing electron density fluctuations in turbulent ionized layers, Proc. IRE 43, 1240 (1955). See also, The spectrum of the electron density fluctuations in the ionosphere, p. 165 in Polar Atmos. Symp., Pt. 2, Ionospheric Sec. (Pergammon Press Inc., New York, N.Y.) 
[19] A. D. Wheelon, Spectrum of turbulent fluctuations produced by convective mixing of gradients, Phys. Rev. 105, 1706 (1957).

[20] A. M. Obukhov, Structure of the temperature field in turbulent flow, Izvest. Akad. Nauk SSSR, Ser. Geog. Geofiz. 13, 58 (1949).

[21] S. Corrsin, On the spectrum of isotropic temperature fluctuations in an isotropic turbulence, J. Appl. Phys. 22, 469 (1951).

[22] R. A. Silverman, Turbulent mixing theory applied to radio scattering, J. Appl. Phys. 27, 699 (1956).

[23] R. Bolgiano, The role of turbulent mixing in scatter propagation, Trans. IRE PGAP AP-6, 159 (1958).

[24] G. K. Batchelor, Small scale variation of convected quantities like temperature in turbulent fluid, I, J. Fluid Mech. 5, 113 (1959).

[25] G. K. Batchelor, I. D. Howells, and A. A. Townsend, Small scale variations of convected quantities like temperature in turbulent fluid, Pt. II, J. Fluid Mech. 5, 134 (1959).

[26] A. D. Wheelon, Diurnal variations of signal level and scattering heights for vhf propagation, J. Geophys. Research 62, 255 (1957).
[27] V. Pineo, Oblique incidence measurements of the heights at which ionospheric scattering of vhf radio waves occur, J. Geophys. Research 61, 165 (1956).

[28] R. C. Kirby, Extreme useful range of vhf transmission by scattering from the lower ionosphere, IRE Conv. Record, Pt. I, p. 112 (1958).

[29] C. Ellyett and H. Leighton, Solar cycle influence on the lower ionosphere and on vhf forward scatter, Proc IRE 46, 1711 (1958)

[30] H. Ekre, K. Endresen, T. Hagfors, B. Landmark, and J. Rodsrud, A study of ionospheric vhf forward scattering at high latitudes, Norweg. Defense Research Establ. Rept. No. 27, Oslo, Norway.

[31] W. J. Bray, J. A. Saxton, R. W. White, and Luscombe, vhf propagation by ionospheric scattering and its application to long-distance communication, Proc. IEE, 1033, p. 236 (Oct. 1955).

[32] S. K. Mitra, The Upper Atmosphere, Calcutta, Asiatic. Soc. Bengal, 2d ed. Asiatic Soc. Monograph Ser. 5, (1952).

(Paper 64D4-62) 